\title{
Rifaximin in the treatment of hepatic encephalopathy
}

This article was published in the following Dove Press journal:

Hepatic Medicine: Evidence and Research

2I December 20II

Number of times this article has been viewed

\section{Maddalena Diana ladevaia \\ Anna Del Prete \\ Claudia Cesaro \\ Laura Gaeta \\ Claudio Zulli \\ Carmelina Loguercio}

Department of Internistica Clinica e Sperimentale, F Magrassi e A Lanzara, Hepatogastroenterology Unit, Second University of Naples, Naples, Italy

Correspondence: Maddalena Diana ladevaia

Via Sergio Pansini, 5 Naples, Italy

Tel +393331647824

Fax +3908 I566 6736

Email maddalenaiadevaia@hotmail.com
Abstract: Hepatic encephalopathy is a challenging complication in patients with advanced liver disease. It can be defined as a neuropsychiatric syndrome caused by portosystemic venous shunting, ranging from minimal to overt hepatic encephalopathy or coma. Its pathophysiology is still unclear, although increased levels of ammonia play a key role. Diagnosis of hepatic encephalopathy is currently based on specific tests evaluating the neuropsychiatric state of patients and their quality of life; the severity of hepatic encephalopathy is measured by the West Haven criteria. Treatment of hepatic encephalopathy consists of pharmacological and corrective measures, as well as nutritional interventions. Rifaximin received approval for the treatment of hepatic encephalopathy in 2010 because of its few side effects and pharmacological benefits. The aim of this work is to review the use and efficacy of rifaximin both in acute and long-term management of hepatic encephalopathy. Treatment of overt hepatic encephalopathy involves management of the acute episode as well as maintenance of remission in those patients who have previously experienced an episode, in order to improve their quality of life. The positive effect of rifaximin in reducing health care costs is also discussed.

Keywords: acute hepatic encephalopathy, recurrent hepatic encephalopathy, rifaximin, lactulose, cost, health-related quality of life

\section{Definition, pathogenesis, and diagnosis of hepatic encephalopathy}

Hepatic encephalopathy is a challenging complication in patients with advanced liver disease. It can be defined as a neuropsychiatric syndrome caused by portosystemic venous shunting, ${ }^{1}$ ranging from minimal to overt hepatic encephalopathy or profound coma. ${ }^{2}$ Minimal hepatic encephalopathy is the mildest form of hepatic encephalopathy, and affected patients have no recognizable clinical symptoms of the disorder, but have subtle abnormalities (elicited by psychometric tests or electrophysiological techniques) in psychomotor speed, visuomotor activity, and response inhibition, as well as slowing of the electroencephalogram and prolongation of cognitive evoked potentials. ${ }^{3}$ Hepatic encephalopathy occurs in approximately $30 \%-45 \%$ of patients with cirrhosis and $10 \%-50 \%$ of patients with transjugular intrahepatic portosystemic shunt, while minimal hepatic encephalopathy affects approximately $20 \%-60 \%$ of patients with liver disease. ${ }^{4}$ Hepatic encephalopathy is an important event in the natural progression of end-stage liver disease; it exerts a negative effect on quality of life for patients and their caregivers, and increases hospitalization rates, with a considerable economic impact. Although the occurrence of episodes of hepatic encephalopathy 
appears to be unrelated to the cause of cirrhosis, ${ }^{5}$ increases in the frequency and severity of such episodes predict an increased risk of death (after the first episode of hepatic encephalopathy the one-year and three-year survival rates are $42 \%$ and $23 \%$, respectively). ${ }^{6}$ According to etiology, a classification system for hepatic encephalopathy disorders was devised by the Working Party at the 1998 World Congress of Gastroenterology in Vienna, Austria. ${ }^{7,8}$

Many details of the pathophysiology leading to encephalopathy remain unclear. The pathogenesis of minimal hepatic encephalopathy is believed to be similar to that of overt hepatic encephalopathy, with increased ammonia levels derived from enteric bacterial flora playing a key role. This leads to astrocyte swelling, which may result in brain edema, increased intracranial pressure, and brain herniation. ${ }^{9}$ Astrocytes are the only cells in the brain that are able to detoxify ammonia. They contain glutamate transporters that are downregulated by the high ammonia level, leading to abnormal glutamatergic neurotransmission. ${ }^{10}$ Moreover, within astrocytes, glutamate combines with ammonia to form glutamine, which is responsible for astrocyte edema, reactive nitrogen, and oxygen species production. ${ }^{11}$ Neurosteroids are synthesized in the brain mainly by astrocytes, and are suggested to play a role in the pathogenesis of hepatic encephalopathy. Ammonia also induces upregulation of peripheral benzodiazepine receptors, which results in increased synthesis of neurosteroids, which bind to gammaaminobutyric acid A receptors and cause neuroinhibition. ${ }^{12}$ Since there is a poor correlation between the plasma ammonia level and the severity of hepatic encephalopathy, participation of concurrent factors has been proposed. Cirrhotic patients have substantial derangements in their gut microecology which has been attributed, at least in part, to a decrease in small intestinal motility. ${ }^{13,14}$ Gupta et al hypothesized that small intestinal bacterial overgrowth might be associated with the presence of minimal hepatic encephalopathy among patients with liver cirrhosis. ${ }^{15}$ Other mechanisms, such as accumulation of manganese into the basal ganglia of the brain (promoting the formation of Alzheimer-type II astrocytosis and Parkinsonian symptoms), ${ }^{16}$ changes in the blood-brain barrier, and neurotransmission disturbances are also present. ${ }^{17}$ Another contributing factor may be systemic inflammatory response syndrome, which derives from ammonia-induced neutrophil dysfunction, leading to a systemic inflammatory response and release of proinflammatory cytokines (such as interleukin-6 and tumor necrosis factor alpha) ${ }^{9}$ which favors astrocyte swelling. ${ }^{18}$ In addition, hyperammonemia is considered to be the main contributor to death in acute liver failure, even if the increase in ammonia is slower and progressive. However, other mechanisms have been implicated, including systemic inflammation, arterial hypotension (resulting from extensive systemic arteriolar vasodilatation), increased cerebral blood flow, and high intracranial pressure. $^{19}$

Diagnosis of minimal hepatic encephalopathy is currently based on neuropsychometric tests, including the number connection test, digit symbol test, and the block design test. In addition, computerized psychometric testing (such as the driving simulator test) and the critical flicker frequency test are increasingly being used. ${ }^{20}$ The sickness impact profile test is used to evaluate patient quality of life. ${ }^{21}$ Moreover, electroencephalography is associated with a decreased mean frequency of electrical activity in the brain (with a diagnostic sensitivity ranging from $43 \%$ to $100 \%$ ), ${ }^{22}$ while magnetic resonance imaging can help to detect low-grade cerebral edema. ${ }^{23}$ Measurement of serum ammonia levels is not recommended routinely, given that the results of the test would not change either the approach to diagnosis or management of hepatic encephalopathy. The severity of hepatic encephalopathy episodes is measured in five progressive stages of impairment known as the West Haven criteria (or Conn score) which ranges from stage 0 (lack of detectable changes in personality or consciousness) to stage 4 (coma). Signs of neuromotor impairment (including hyperreflexia, rigidity, myoclonus, and asterixis) are measured using the asterixis severity scale. ${ }^{?}$

In the management of hepatic encephalopathy, the physician should follow the "three-steps" algorithm:

- Rule out other potential causes of encephalopathy that often occur in patients with cirrhosis (eg, subdural hematoma because of the higher risk of falls and coagulopathies, and side effects of medication causing dysfunction of the liver, ie, the site of first-pass metabolism) ${ }^{24}$

- Identify and correct precipitating factors (gastrointestinal hemorrhage, dehydration, dietary protein overload, infection) present in $97 \%$ of patients with episodic hepatic encephalopathy and in more than $70 \%$ with persistent hepatic encephalopathy

- Trial empiric treatment (as discussed below); a rapid response to which confirms the diagnosis of hepatic encephalopathy, whereas a lack of response within 72 hours indicates that further treatment or causes should be considered. ${ }^{8}$

\section{Treatment of hepatic encephalopathy}

Treatment of an hepatic encephalopathy episode includes corrective measures, nutritional intervention, and 
pharmacological therapy. As already discussed, identification and correction of precipitating factors is considered firstline therapy, and includes bleeding control, correction of metabolic disorders, and treatment of infection. Moreover, it is necessary to maintain adequate nutrition, with an energy intake of 35-40 kcal/kg/day and protein intake of $1.2-1.5 \mathrm{~g} / \mathrm{kg} / \mathrm{day},{ }^{25}$ and the diet of patients with cirrhosis should be supplemented with branched-chain amino acids and protein vegetables once hepatic encephalopathy has developed. ${ }^{26,27}$

The aim of pharmacological treatment is both to reduce ammonia production and to favor its fixation and excretion. The clinician armamentarium against hepatic encephalopathy is limited; medical treatment for hepatic encephalopathy has not changed over the last 30 years, not because the available drugs are highly effective and evidencebased treatment options for hepatic encephalopathy, but because of lack of knowledge concerning the pathogenesis of hepatic encephalopathy, its clinical heterogeneity, and variable assessment of its severity. ${ }^{28}$ Treatment of hepatic encephalopathy traditionally includes lactulose, neomycin, or metronidazole, and, most recently, rifaximin. The current standard of care for patients with hepatic encephalopathy includes lactulose and rifaximin, which is associated with improvement in mental status; however, because precipitating factors are simultaneously being corrected, it is difficult to establish the true reason for improvement. ${ }^{29}$ Lactulose is a nonabsorbable synthetic disaccharide, which reaches the colon unaltered where it has a cathartic effect and is catabolized by colonic bacterial flora to produce lactic acid and acetic acid. ${ }^{30}$ The resulting acidic colonic environment inhibits growth of ammoniagenic coliform bacteria and favors the conversion of ammonia into nonadsorbable ammonium. Lactulose can be administered orally through a nasogastric tube to a comatose or unresponsive patient or rectally through an enema. The usual oral dose is about $15-30 \mathrm{~mL}$ twice daily to induce 2-3 soft bowel movements daily. However, side effects of this drug, including an excessively sweet taste and gastrointestinal side effects, such as bloating, flatulence, and severe diarrhea, possibly leading to dehydration, often result in noncompliance. ${ }^{31}$ For patients who cannot tolerate or do not respond to therapy with lactulose, oral antibiotics, such as neomycin, vancomycin, paromomycin, and metronidazole, are recommended as an alternative therapy. These are all effective in the treatment of hepatic encephalopathy, but their serious side effects (ototoxicity and nephrotoxicity for neomycin and paromomycin and peripheral neuropathy for metronidazole) limit their use as first-line or long-term therapy. ${ }^{33}$ Originally approved for the management of traveler's diarrhea, rifaximin received approval from the US Food and Drug Administration in March 2010 for the treatment of hepatic encephalopathy because of its few side effects and pharmacological benefits in reducing the risk of recurrence of overt hepatic encephalopathy.

\section{Rifaximin: pharmacology, pharmacokinetics, and mode of action}

Rifaximin is a semisynthetic, gut-selective, and nonabsorbable oral antibiotic, derived from rifamycin and a structural analog of rifampin. It acts locally in the gastrointestinal tract, with systemic adverse effects that are similar to placebo. It is active against a variety of aerobic and anaerobic Gram-positive and Gram-negative organisms, as well as protozoal infections. ${ }^{33}$ In vitro data indicate that the susceptibility of Gram-positive organisms to rifaximin is greater than that of Gramnegative organisms. Rifaximin possesses good inhibitory activity against species of Staphylococci, Streptococci, and Enterococci as well as Bacillus cereus, Moraxella catarrhalis, and Haemophilus influenzae. Activity is lower against species of Enterobacteriaceae, Pseudomonads, Acinetobacter, and Helicobacter, and conflicting data exist for activity against Bacteroides spp. Like other rifamycin antibacterial agents, rifaximin acts on the [beta] subunit of the deoxyribonucleic acid (DNA)-dependent ribonucleic acid (RNA) polymerase enzyme in bacteria to inhibit bacterial RNA synthesis. Comparable antimicrobial activity was seen between rifaximin and other antimicrobials for Escherichia coli. In addition, activity against Clostridium difficile was comparable with that of metronidazole and vancomycin. ${ }^{34}$ Importantly, administration of rifaximin $800 \mathrm{mg}$ /day to 10 healthy volunteers for 5 days resulted in a marked reduction in the numbers of some enteric bacteria, which subsequently normalized within 1-2 weeks of discontinuing therapy. ${ }^{35}$ Pharmacokinetic parameters of rifaximin were compared between healthy subjects and patients with Child-Pugh class A, B, and C cirrhosis. Patients with more severe hepatic impairment (class $\mathrm{C}$ cirrhosis) had increased levels of systemic exposure to rifaximin than those with class A or B cirrhosis. Therefore, caution is recommended when using rifaximin in patients with advanced cirrhosis. ${ }^{36}$

\section{Dosage and administration}

The recommended rifaximin dosage for adults and children older than 12 years is $10-15 \mathrm{mg} / \mathrm{kg} /$ day, while a higher daily dose of $20-30 \mathrm{mg} / \mathrm{kg} /$ day is suggested in younger children. 
Treatment duration should not exceed 7 days in children. Rifaximin should not be administered to patients with evidence of serious intestinal ulcerative lesions or obstruction. Rifaximin dosing can be approached in two broad ways, ie, cyclical or continuous. In Italy, cyclical dosing is preferred, and several clinical trials have shown benefit from treatment with rifaximin for 2 weeks per month. ${ }^{37}$ The alternative is daily therapy with rifaximin, as is currently being used in the US. There are possible advantages and disadvantages to each approach; cyclical therapy reduces cost and antibiotic exposure, but adherence to the schedules may be difficult. Continuous therapy is more expensive and could have the potential to increase resistance to rifaximin. The daily dose most studied, whether cyclical or continuous, is $1200 \mathrm{mg}$, although the most recent trial used $1100 \mathrm{mg} /$ day. ${ }^{38}$

\section{Resistance}

The primary mechanism for developing resistance to rifaximin is chromosomal alteration of the drug target, ie, DNA-dependent RNA polymerase, which is in contrast with the plasmid-mediated resistance that affects other antibiotics. Therefore, resistance to rifaximin is not transmissible easily between bacteria. ${ }^{39}$ In vitro data show that the organisms which most commonly develop resistance to rifaximin are aerobic Gram-positive cocci; anaerobic conditions are not conducive to selecting resistant mutants. In addition, Gram-negative organisms, such as E. coli, have not been demonstrated to develop resistance to rifaximin after 3-5 days of therapy. ${ }^{40}$ Because rifaximin lacks systemic absorption, drug concentrations stay well above the minimum inhibitory concentration of pathogens, thus avoiding subtherapeutic drug concentrations in the intestine. In addition, the intestinal lumen is predominantly an anaerobic environment, theoretically limiting selection of isolates resistant to rifaximin. However, despite these advantages, and potentially due to the structural relationship between rifaximin and other rifamycins, resistance rates for Enterococci, Bacteroides, Clostridium, and Enterobacteriaceae range between 30\% to $90 \%$ after 5 days of treatment. After rifaximin is stopped, these resistant strains tend to disappear within 1-12 weeks. ${ }^{41}$

\section{Drug interactions}

Rifaximin undergoes efflux through P-glycoprotein and does not have significant interactions with other substrates for P-glycoprotein, such as digoxin. The ability of rifaximin to induce cytochrome P450 (CYP)3A4 is half that of rifampin in studies in vitro. Two studies suggested a lack of in vivo interaction between rifaximin and a CYP3A4 substrate. ${ }^{42,43}$ Therefore, no dose adjustment is recommended when rifaximin is administered with other drugs.

\section{Potential indications}

The virtual lack of systemic absorption after oral administration indicates a potential role for rifaximin in localized conditions mediated by susceptible bacteria within the gastrointestinal tract. Rifaximin has been evaluated in the symptomatic management of hepatic encephalopathy, infectious diarrhea, and diverticular disease, and as prophylaxis against postoperative complications following colorectal surgery.

\section{Safety and tolerability}

Rifaximin is generally well tolerated, and has a better profile in terms of side effects when compared with other systemic antibiotics. Several studies showed that rifaximin was better tolerated than lactulose in the treatment of hepatic encephalopathy and had a placebo-like tolerability profile in patients in hepatic encephalopathy remission. The risk of bacterial resistance appeared to be low; plasma levels of rifaximin are negligible, and bacteria outside the gastrointestinal tract are not exposed to appreciable selective pressure. In addition, the mechanism of resistance to rifaximin is by chromosomal alteration in DNA-dependent RNA polymerase, which is in contrast with the clinically significant plasmid-mediated resistance that affects other antibiotics. Therefore, the resistance to rifaximin is not transmissible easily between bacteria. However, the clinical relevance of this resistance, especially for long-term therapy, needs to be studied. ${ }^{29}$ In a study conducted by Bass et al, ${ }^{37} \mathrm{C}$. difficile infection was reported in two patients on rifaximin. They both had several concurrent risk factors, including advanced age, numerous recent hospitalizations, multiple courses of antibiotics, and use of proton pump inhibitors. Clinicians should be vigilant against $C$. difficile in patients with cirrhosis receiving long-term therapy with rifaximin, because the infection has a poor prognosis in this class of patients and diarrhea in these patients is often attributed to concomitant use of lactulose, leading to a delayed diagnosis. ${ }^{29} \mathrm{We}$ should not forget that rifaximin, as well as others, can cause alteration of the gut flora.

\section{Rifaximin in acute hepatic encephalopathy}

The aim of this work is to review the efficacy of rifaximin in both the acute and long-term management of hepatic encephalopathy. Management of overt hepatic 
encephalopathy consists of both treatment of the acute episode and maintenance of remission of patients who have previously experienced an episode of hepatic encephalopathy, in order to improve their quality of life. Both lactulose and rifaximin have received approval from the US Food and Drug Administration for this purpose. Several studies have been conducted to support the use of rifaximin instead of or in addition to lactitol/lactulose in the treatment of acute hepatic encephalopathy. ${ }^{43-54}$

Mas et $\mathrm{al}^{43}$ conducted a prospective, randomized, double-blind, double-dummy, controlled clinical study in order to assess the efficacy and the safety of rifaximin in comparison with lactitol for the treatment of cirrhotic patients with grade I-III acute or recurrent hepatic encephalopathy. One hundred and three patients were randomized to receive rifaximin $(\mathrm{n}=50,1200 \mathrm{mg} /$ day $)$ or lactitol $(\mathrm{n}=53$, $60 \mathrm{~g} /$ day) for 5-10 days. Both groups were comparable with regard to demographic data and characteristics of the hepatic encephalopathy episode. Changes in portosystemic encephalopathy (index on entry and at the end of the study) were used to evaluate the efficacy of the two therapies. The investigators found that both therapies were effective, but a greater effect on electroencephalographic abnormalities and ammonia levels (the two components of the portosystemic encephalopathy index) was observed in the rifaximin group.

Loguercio et $\mathrm{al}^{44}$ enrolled 40 patients with chronic hepatic encephalopathy (I-II severity). Mental state, asterixis, number connection test, and arterial blood ammonia levels were used to assess hepatic encephalopathy. Patients were randomly assigned to treatments with rifaximin, lactitol, and rifaximin plus lactitol. All treatments were continued for 15 days for three cycles, with a 15-day washout between treatments. They concluded that all treatments reduced hepatic encephalopathy, but with different efficacy; patients on rifaximin and those on rifaximin plus lactitol documented faster improvement in hepatic encephalopathy, higher percentages of normalized mental state and performance on the number connection test, faster improvement of asterixis, and longer persistence of normal ammonia levels than patients on lactitol.

Some years before, Bucci and Palmieri ${ }^{45}$ had already reported an improvement in symptoms of portosystemic encephalopathy in patients randomly assigned to two treatment groups (rifaximin $1200 \mathrm{mg}$ /day and lactulose $30 \mathrm{~g} /$ day, both for 15 days). Improvement was correlated with reduction in levels of serum ammonia concentrations, recorded after only 3 days of treatment. Tolerability of treatment with rifaximin was decidedly higher with respect to lactulose.

\section{Rifaximin in reducing risk of recurrent hepatic encephalopathy}

Lactulose and rifaximin are the most popular choices for ongoing therapy in patients who have experienced an episode of hepatic encephalopathy. However, adherence of patients to long-term therapy with lactulose is limited by its adverse effects. Therefore, rifaximin is emerging as a first-line therapy to reduce the incidence of recurrent hepatic encephalopathy. The efficacy and safety of rifaximin in maintaining remission of hepatic encephalopathy over 6 months in patients at high risk were assessed by Bass et $\mathrm{al}^{37}$ who conducted a randomized, double-blind, placebocontrolled, multicenter, multinational Phase III trial. This study included patients with at least two episodes of overt hepatic encephalopathy (Conn score $\geq 2$ ) associated with hepatic cirrhosis during the previous 6 months. At enrollment they had recovered from hepatic encephalopathy and had a model for end-stage liver disease (MELD) score $\leq 25$. Patients were excluded if they had had a planned liver transplantation within one month, conditions known to precipitate hepatic encephalopathy, chronic renal disease, respiratory insufficiency, anemia, or electrolyte abnormalities. A transjugular intrahepatic portosystemic shunt could be present, but it could not have been placed or revised within the past 3 months. Patients could not be on daily prophylactic antibiotic therapy at the outset. Two-hundred and ninety-nine patients were randomized to rifaximin $(n=140)$ or placebo $(n=159)$ for 6 months or until they discontinued the study because of a breakthrough episode of hepatic encephalopathy or another reason. Lactulose was permitted. The objectives were to assess time to first breakthrough of overt hepatic encephalopathy, to first hepatic encephalopathyrelated hospitalization, and to any increase from baseline in Conn score, in asterixis grade, mean change in baseline in fatigue domain scores on the chronic liver disease questionnaire (CLQD) at end of treatment, and mean change from baseline in venous ammonia concentration at end of treatment. A breakthrough episode of hepatic encephalopathy was reported for $22.1 \%$ of patients receiving rifaximin and $45.9 \%$ of patients receiving placebo $(P<0.001,95 \%$ confidence interval [CI]: 0.28-0.64). Regarding the second endpoint of the study, a total of $13.6 \%$ of the patients in the rifaximin group had a hospitalization involving hepatic encephalopathy as compared with $22.6 \%$ of patients in the 
placebo group. The incidence of adverse events reported during the study was similar in the two groups (two patients in the rifaximin group developed a $C$. difficile infection but this was not related to the antibiotic per se according to the authors). The innovation of this study was the examining of the protective effect of rifaximin against breakthrough episodes of hepatic encephalopathy rather than its effect in the treatment of overt acute symptoms. Moreover, rifaximin reduced the risk of hospitalization for hepatic encephalopathy. Limitations of this study include a short duration of treatment and involvement of the sponsor in the protocol design, data analysis, and revision of the manuscript. The main questions that remain to be answered are whether rifaximin has the same efficacy in monotherapy (since more than $90 \%$ of patients were on combined therapy with lactulose) in more severe cases of hepatic encephalopathy (the majority of patients had a MELD score $\leq 19$ ) and whether it exerts any long-term effects on gut flora.

\section{Influence of rifaximin on economic burden of hepatic encephalopathy}

Approximately 5.5 million persons in the US have hepatic cirrhosis, and most of them have a lifetime risk of developing an episode of hepatic encephalopathy. Although the total direct and indirect costs of hepatic encephalopathy have not been formally quantified, data from the Healthcare Cost and Utilization Project suggest that hepatic encephalopathy-related hospitalizations are associated with substantial health care costs. In 2003, there were over 40,000 patients hospitalized in the US for a primary diagnosis of hepatic encephalopathy, resulting in total charges of approximately US\$932 million. Furthermore, trends over the past 10 years suggest that the burden of hepatic encephalopathy is increasing, as indicated by increases in hospital admissions and higher charges per stay. Moreover, hepatic encephalopathy imposes a formidable burden on families, because patients are incapable of selfcare. $^{1,4,55-57}$

Neff et $a^{55}$ performed a retrospective review in which they discussed the cost comparison between standard therapy of lactulose and non-nonabsorbable therapy in liver transplanted patients presenting with stage II hepatic encephalopathy. In this study, the importance of cost-analysis as opposed to drug price when analyzing health care expenditures is reasserted. In fact, the price analysis between rifaximin and lactulose strongly favors the second one, but cost analysis (in terms of decreasing health care costs and hospitalizations) shows a favorable advantage for rifaximin. These investigators concluded that therapies for hepatic encephalopathy should be directed toward the prevention of hospitalization (which involves the use of rifaximin).

Similarly, Leevy and Phillips ${ }^{56}$ compared frequency and duration of hepatic encephalopathy-related hospitalizations during rifaximin versus lactulose treatment. Treatment of hepatic encephalopathy with rifaximin was associated with lower hospitalization frequency and duration, lower hospital charges, better clinical status, and fewer adverse events. The beneficial role of rifaximin in reducing the risk, number, and duration of hospitalizations for hepatic encephalopathy was also confirmed by Mantry and Munsaf. ${ }^{57}$ Therefore, this study introduced a new concept, ie, treatment of hepatic encephalopathy with rifaximin may also provide benefit by reducing risk of hospitalization for conditions other than hepatic encephalopathy, such as cirrhosis-related complications (portal hypertension, variceal bleeding, spontaneous bacterial peritonitis), which may be related to a reduction in bacterial overgrowth in the gastrointestinal tract and subsequent reduction in translocation of bacteria from intestinal lumen into the bloodstream. ${ }^{58}$

\section{Influence of rifaximin on health-related quality of life}

Hepatic encephalopathy predisposes patients to impaired quality of life as a result of repeated hospitalizations, severity of liver disease, and complications of cirrhosis, such as recurrent overt hepatic encephalopathy or ongoing minimal hepatic encephalopathy. ${ }^{59}$ Overt hepatic encephalopathy negatively affects both physical and mental aspects of quality of life, whereas subclinical encephalopathy affects mainly the mental aspects, independently of severity of liver disease, ${ }^{60}$ with cognitive dysfunction and difficulties in driving and navigation (patients with minimal hepatic encephalopathy have an increased risk of driving offences). ${ }^{61}$ The chronic liver disease questionnaire is a validated health-related quality of life (HRQL) instrument to measure longitudinal change over time in patients with chronic liver disease. It includes 29 items in six domains, ie, abdominal symptoms, fatigue, systemic symptoms, activity, emotional function, and worry. Rifaximin, as well as lactulose, are known to improve HRQL. 2,62,65 Sanyal et $\mathrm{l}^{62}$ conducted a double-blind, placebo-controlled study to evaluate the effect of rifaximin on HRQL in 219 cirrhotic patients with hepatic encephalopathy. They were randomized to receive rifaximin $550 \mathrm{mg}$ twice daily or 
placebo for 6 months. The CLDQ was administered to patients every 4 weeks and time to occurrence of hepatic encephalopathy breakthrough was recorded. They found that rifaximin significantly improved HRQL in patients with cirrhosis and recurrent hepatic encephalopathy. Moreover, although not a measure of HRQL, the reduction in risk of hospitalization with rifaximin had a positive impact on HRQL for these patients, given that a lower HRQL may predict recurrence of hepatic encephalopathy. As showed by Sidhu et al, ${ }^{64}$ rifaximin also improves psychometric performance in patients with myalgic encephalopathy, as assessed by the sickness impact profile questionnaire, which interrogates 12 domains of daily functioning. ${ }^{22}$ Moreover, they confirmed the ability of rifaximin to reverse myalgic encephalopathy and to reduce the mean number of abnormal neuropsychometric tests administered to patients. A recent randomized, placebo-controlled trial ${ }^{65}$ evaluated whether rifaximin improved driving performance in patients with myalgic encephalopathy using a driving simulator. The authors found greater improvements in the rifaximin group than placebo group in avoiding driving errors, speeding, and illegal turns. They similarly improved their cognitive performance and psychosocial dimension as assessed by the sickness impact profile questionnaire.

\section{Conclusion}

Although proven to be effective in treating episodes of hepatic encephalopathy, in preventing recurrence of hepatic encephalopathy (and so reducing hospitalization rate), and in improving HRQL in cirrhotic patients, rifaximin is still regarded as a second-line therapy because of its cost (higher than that of lactulose) and its ability to alter gut flora. Lactulose in monotherapy can prevent recurrent hepatic encephalopathy episodes. Only short-term studies using rifaximin as first-line therapy have been conducted in the past. Bajaj and Riggio ${ }^{29}$ suggested that rifaximin may become first-line therapy in hepatic encephalopathy with the support of long-term and head-to-head studies. Only one study ${ }^{66}$ directly compared lactulose and rifaximin in preventing hepatic encephalopathy in patients who underwent transjugular intrahepatic portosystemic shunt, and both therapies showed no efficacy. Similarly Bass et $\mathrm{al}^{37}$ used rifaximin in patients who had already failed on lactulose. The role of rifaximin in patients with a MELD score $>19$ has not been investigated. In conclusion, the key question of "use of rifaximin instead of or in addition to lactulose" remains to be answered.

\section{Disclosure}

The authors report no conflicts of interest in this work.

\section{References}

1. Munoz SJ. Hepatic encephalopathy. Med Clin North Am. 2008;92: 795-812.

2. Prasad S, Dhiman RK, Duseja A, Chawla YK, Sharma A, Agarwal R. Lactulose improved cognitive function and health related quality of life in patients with cirrhosis who have minimal hepatic encephalopathy. Hepatology. 2007;45:549-559.

3. Amodio P, Montagnese S, Gatta A, et al. Characteristics of minimal hepatic encephalopathy. Metab Brain Dis. 2004;19:253-267.

4. Poordad FF. The burden of hepatic encephalopathy. Aliment Pharmacol Ther. 2007;(25 Supp1 1):3-9.

5. Kalaitzakis E, Josefsson A, Bjornsson E. Type and etiology of liver cirrhosis are not related to the presence of hepatic encephalopathy on health-related quality of life: a cross sectional study. $B M C$ Gastroenterol. 2008;8:46.

6. DeFrances CJ, Cullen KA, Kozak LJ. National Hospital Discharge Survey: 2005 annual summary with detailed diagnosis and procedure data. Vital Health Stat. 2007;13:1-209.

7. Ferenci P, Lockwood A, Mullen K, Tarter R, Weissenborn K, Blei AT; Members of the Working Party. Hepatic encephalopathy - definition, nomenclature, diagnosis, and quantification: Final Report of the Working Party at the 11th World Congresses of Gastroenterology, Vienna, Austria, 1998.

8. Prakash R, Mullen KD. Mechanisms, diagnosis and management of hepatic encephalopathy. Nat Rev Gastroenterol Hepatol. 2010;7: $515-525$.

9. Shawcross DL, Shabbir SS, Taylor NJ, Hughes RD. Ammonia and the neutrophil in the pathogenesis of hepatic encephalopathy in cirrhosis. Hepatology. 2010;51:1062-1069.

10. Knecht K, Michalak A, Rose C, Rothstein JD, Butterworth RF. Decreased glutamate transporter (GLT-1) expression in frontal cortex of rats with acute liver failure. Neurosci Lett. 1997;229:201-203.

11. Albrecht J, Norenberg MD. Glutamine: a Trojan horse in ammonia neurotoxicity. Hepatology. 2006;44:788-794.

12. Ahboucha S, Butterworth RF. The neurosteroid system: implication in the pathophysiology of hepatic encephalopathy. Neurochem Int. 2008;52:575-587.

13. Chesta J, Defilippi C, Defilippi C. Abnormalities in proximal small bowel motility in patients with cirrhosis. Hepatology. 1993;17:828-832.

14. Chang CS, Chen GH, Lien HC, Yeh HZ. Small intestine dysmotility and bacterial overgrowth in cirrhotic patients with spontaneous bacterial peritonitis. Hepatology. 1998;28:1187-1190.

15. Gupta A, Dhiman RK, Kumari S, et al. Role of small intestinal bacterial overgrowth and delayed gastrointestinal transit time in cirrhotic patients with minimal hepatic encephalopathy. J Hepatol. 2010;53: 849-855.

16. Krieger D, Krieger S, Jansen O, Gass P, Theilmann L, Lichtnecker H. Manganese and chronic hepatic encephalopathy. Lancet. 1995;346: 270-274.

17. Mas A. Hepatic encephalopathy: from pathophysiology to treatment. Digestion. 2006;(73 Suppl 1):86-93.

18. Blei AT. Infection, inflammation and hepatic encephalopathy, synergism redefined. J Hepatol. 2004;40:327-330.

19. Bjerring PN, Eefsen M, Hansen BA, Larsen FS. The brain in acute liver failure. A tortuous path from hyperammonemia to cerebral edema. Metab Brain Dis. 2009;24:5-14.

20. Butterworth RF. Rifaximin and minimal hepatic encephalopathy. Am J Gastroenterol. 2011;106:317-318.

21. Bergner M, Bobbitt RA, Cartner WB, et al. The sickness impact profile: development and final revision of a health status measure. Med Care. 1981;19:787-805. 
22. Montagnese S, Amodio P, Morgan MY. Methods for diagnosing hepatic encephalopathy in patients with cirrhosis: a multidimensional approach. Metab Brain Dis. 2004;19:281-312.

23. Rovira A, Alonso J, Cordoba J. MR imaging findings in hepatic encephalopathy. AJNR Am J Neuroradiol. 2008;29:1612-1621.

24. Tsuji H, Kashiwagi M, Ikeda K, et al. A case of liver cirrhosis associated with chronic subdural hematoma and hepatic encephalopathy. Fukuoka Igaku Zasshi. 1991;82:528-532. Japanese.

25. Cordoba J, Lopez-Hellin J, Planas M, et al. Normal protein diet for episodic hepatic encephalopathy: results of a randomized study. J Hepatol. 2004;41:38-43.

26. Muto Y, Sato S, Watanabe A, et al. Effects of oral branched-chain amino acid granules on event-free survival in patients with liver cirrhosis. Clin Gastroenterol Hepatol. 2005;3:705-713.

27. Amodio P, Caregaro L, Pattenò E, Marcon M, Del Piccolo F, Gatta A. Vegetarian diets in hepatic encephalopathy: facts or fantasies? Dig Liver Dis. 2001;33:492-500.

28. Leise MD, Kim WR. Rifaximin in hepatic encephalopathy: is an ounce of prevention worth a pretty penny? Gastroenterology. 2010;139: 1416-1418.

29. Bajaj JS, Riggio O. Drug therapy: rifaximin. Hepatology. 2010;52: 1484-1488.

30. Al Sibae MR, McGuire BM. Current trends in the treatment of hepatic encephalopathy. Ther Clin Risk Manag. 2009;5:617-626.

31. Als-Nielsen B, Gluud LL, Gluud C. Non-absorbable disaccharides for hepatic encephalopathy: systematic review of randomised trials. $B M J$. 2004;328:1046.

32. Maddrey WC. Role of antibiotics in the management of hepatic encephalopathy. Rev Gastroenterol Disord. 2005;(5 Suppl 1):S3.

33. Gillis JC, Brogden RN. Rifaximin: a review of its antibacterial activity, pharmacokinetic properties and therapeutic potential in conditions mediated by gastrointestinal bacteria. Drugs. 1995;49:67-84.

34. Marchese A, Salerno A, Pesce A, Debbia EA, Schito GC. In vitro activity of rifaximin, metronidazole and vancomycin against Clostridium difficile and the rate of selection of spontaneously resistant mutants against representative anaerobic and aerobic bacteria, including ammoniaproducing species. Chemotherapy. 2000;46:253-266.

35. Testa R, Eftimiadi C, Sukkar GS, et al. A non-absorbable rifamycin for treatment of hepatic encephalopathy. Drugs Exp Clin Res. 1985;11: 387-392.

36. Alcorn J. Review: rifaximin is equally or more effective than other antibiotics and lactulose for hepatic encephalopathy. ACP J Club. 2008; 149:11.

37. Bass NM, Mullen KD, Sanyal A, et al. Rifaximin treatment in hepatic encephalopathy. $N$ Engl J Med. 2010;362:1071-1081.

38. Spratt BG. Resistance to antibiotics mediated by target alterations. Science. 1994;264:388-393.

39. DuPont HL, Jiang ZD. Influence of rifaximin treatment on the susceptibility of intestinal Gram-negative flora and enterococci. Clin Microbiol Infect. 2004;10:1009-1011.

40. De Leo C, Eftimiadi C, Schito GC. Rapid disappearance from the intestinal tract of bacteria resistant to rifaximin. Drugs Exp Clin Res. 1986;12:979-981.

41. Trapnell CB, Connolly M, Pentikis H, et al. Absence of effect of oral rifaximin on the pharmacokinetics of ethinyl estradiol/norgestimate in healthy females. Ann Pharmacother. 2007;41:222-228.

42. Pentikis HS, Connolly M, Trapnell CB, et al. The effect of multiple-dose, oral rifaximin on the pharmacokinetics of intravenous and oral midazolam in healthy volunteers. Pharmacotherapy. 2007;27: 1361-1369.

43. Mas A, Rodés J, Sunyer L, et al. Comparison of rifaximin and lactitol in the treatment of acute hepatic encephalopathy: results of a randomized, double-blind, double-dummy, controlled clinical trial. J Hepatol. 2003;38:51-58.

44. Loguercio C, Federico A, De Girolamo V, Ferrieri A, Del Vecchio Blanco C. Cyclic treatment of chronic hepatic encephalopathy with rifaximin. Results of a double-blind clinical study. Minerva Gastroenterol Dietol. 2003;49:53-62.
45. Bucci L, Palmieri GC. Double-blind, double-dummy comparison between treatment with rifaximin and lactulose in patients with medium to severe degree hepatic encephalopathy. Curr Med Res Opin. 1993;13:109-118.

46. Massa P, Vallerino E, Dodero M. Treatment of hepatic encephalopathy with rifaximin: double-blind, double dummy study versus lactulose. Eur J Clin Res. 1993;4:7-18.

47. Pedretti G, Calzetti C, Missale G, Fiaccadori F. Rifaximin versus neomycin on hyperammoniemia in chronic portal systemic encephalopathy of cirrhotics. A double-blind, randomized trial. Ital J Gastroenterol. 1991;23:175-178

48. Sama C, Morselli-Labate AM, Pianta P, Lambertini L, Berardi S, Martini G. Clinical effects of rifaximin in patients with hepatic encephalopathy intolerant or nonresponsive to previous lactulose treatment: An open labeled study. Curr Ther Res. 2004;65: 413-422.

49. Williams R, James OF, Warnes TW, Morgan MY. Evaluation of the efficacy and safety of rifaximin in the treatment of hepatic encephalopathy: a double-blind, randomized, dose-finding multicentre study. Eur J Gastroenterol Hepatol. 2000;12:203-208.

50. Venturini I, Ferrieri A, Farina F, et al. Evaluation of rifaximin, placebo and lactulose in reducing the levels of benzodiazepine-like compounds in patients with liver cirrhosis: a pilot study. Drugs Exp Clin Res. 2005;31: 161-168.

51. Paik YH, Lee KS, Han KH, et al. Comparison of rifaximin and lactulose for the treatment of hepatic encephalopathy: a prospective randomized study. Yonsei Med J. 2005;46:399-407.

52. Riggio O, Nicolao F, Angeloni S, et al. Intractable hepatic encephalopathy after tips with polytetrafluoroethylene-covered stent graft. Scand J Gastroenterol. 2003;38:570-572.

53. Miglio F, Valpiani D, Rossellini SR, Ferrieri A. Rifaximin, a nonabsorbable rifamycin, for the treatment of hepatic encephalopathy. A double-blind, randomised trial. Curr Med Res Opin. 1997;13: 593-601.

54. Di Piazza S, Gabriella Filippazzo M, Valenza LM, et al. Rifaximine versus neomycin in the treatment of portosystemic encephalopathy. Ital J Gastroenterol. 1991;23:403-407.

55. Neff GW, Kemmer N, Zacharias VC, et al. Analysis of hospitalizations comparing rifaximin versus lactulose in the management of hepatic encephalopathy. Transplant Proc. 2006;38:3552-3555.

56. Leevy CB, Phillips JA. Hospitalizations during the use of rifaximin versus lactulose for the treatment of hepatic encephalopathy. Dig Dis Sci. 2007;52:737-741.

57. Mantry PS, Munsaf S. Rifaximin for the treatment of hepatic encephalopathy. Transplant Proc. 2010;42:4543-4547.

58. Cheruvattath R, Balan V. Infections in patients with end-stage liver disease. J Clin Gastroenterol. 2007;41:403-411.

59. Marchesini G, Bianchi G, Amodio P, et al; Italian Study Group for Quality of Life in Cirrhosis. Factors associated with poor health-related quality of life of patients with cirrhosis. Gastroenterology. 2001;120: $170-178$.

60. Arguedas MR, DeLawrence TG, McGuire BM. Influence of hepatic encephalopathy on health-related quality of life in patients with cirrhosis. Dig Dis Sci. 2003;48:1622-1626.

61. Kircheis G, Knoche A, Hilger N, et al. Hepatic encephalopathy and fitness to drive. Gastroenterology. 2009;137:1706-1715.

62. Sanyal A, Younossi ZM, Bass NM, et al. Randomised clinical trial: rifaximin improves health related quality of life in cirrhotic patients with hepatic encephalopathy - a double-blind placebo controlled study. Aliment Pharmacol Ther. 2011;34:853-861.

63. Kalaitzakis E, Bjornsson E. Lactulose treatment for hepatic encephalopathy, gastrointestinal symptoms, and health-related quality of life. Hepatology. 2007;46:949-950.

64. Sidhu SS, Goyal O, Mishra BP, Sood A, Chhina RS, Soni RK. Rifaximin improves psychometric performance and health-related quality of life in patients with minimal hepatic encephalopathy (the RIME Trial). Am J Gastroenterol. 2011;106:307-316. 
65. Bajaj JS, Heuman DM, Wade JB, et al. Rifaximin improves driving simulator performance in a randomized trial of patients with minimal hepatic encephalopathy. Gastroenterology. 2011;140:478.e1-487.e1.
66. Riggio O, Masini A, Efrati C, et al. Pharmacological prophylaxis of hepatic encephalopathy after transjugular intrahepatic portosystemic shunt: a randomized controlled study. J Hepatol. 2005;42:674-679.

\section{Publish your work in this journal}

Hepatic Medicine: Evidence and Research is an international, peerreviewed, open access journal covering all aspects of adult and pediatric hepatology in the clinic and laboratory including the following topics: Pathology, pathophysiology of hepatic disease; Investigation and treatment of hepatic disease; Pharmacology of drugs used for the treatment of hepatic disease. Issues of patient safety and quality of care will also be considered. The manuscript management system is completely online and includes a very quick and fair peer-review system, which is all easy to use. Visit http://www.dovepress.com/ testimonials.php to read real quotes from published authors.

Submit your manuscript here: http://www.dovepress.com/hepatic-medicine-evidence-and-research-journal 